\title{
Sources of information in times of health crisis: evidence from Turkey during COVID-19
}

\author{
Ekmel Geçer ${ }^{1}$ (i) $\cdot$ Murat Yıldırım $^{2,3}$ (1) $\cdot$ Ömer Akgül $^{1}$ (i)
}

Received: 6 June 2020 / Accepted: 5 October 2020 / Published online: 13 October 2020

(C) Springer-Verlag GmbH Germany, part of Springer Nature 2020

\begin{abstract}
Aim Media as a source of information can shape public opinion regarding the COVID-19 response. Identifying how and where people seek information during the COVID-19 outbreak is vital to convey the most effective message about managing the COVID-19 crisis. The purpose of this study was to determine the sources of information and investigate the role of various demographic factors - age, gender, educational attainment and perceived economic level—on sources of information.

Subject and methods An online survey $(n=4624)$ was conducted on Turkish public during the early stages of the COVID-19. Results The results showed that internet journalism and social media were the most preferable sources of information. Higher age, educational attainment and economic level were related to higher levels of seeking information from TV, newspaper, internet journalism and informative meetings. Females obtained information more from their friends and family and social media than males. High school graduates or below watched more TV and obtained less information from internet journalism, while university graduates sought information from their families and friends, and postgraduates attended informative meetings and read newspapers. People with medium and high economic status, respectively, watched more TV and read more newspapers, while people with low socioeconomic status attended informative meetings less.

Conclusion In sum, this study provides evidence that a source of information might be influenced by demographic factors. Researchers and policymakers can use a source of information to develop crisis-response strategies by considering variations in the demographic factors.
\end{abstract}

Keywords COVID-19 · Coronavirus · Source of information · Demographic factors · Turkey

The world is currently under the threat of the fast spreading novel coronavirus disease (COVID-19). In December 2019, the World Health Organization (WHO 2020a) reported many pneumonia cases from Wuhan city in China. On 12 December 2019, the first case of the COVID-19 epidemic was reported with unexplained pneumonia, and on December 31, 2019, 27 viral pneumonia cases, with seven being severe, were

Murat Yildırım

muratyildirim@agri.edu.tr

1 Department of Psychology, Hamidiye Faculty of Life Sciences, University of Health Sciences, Istanbul, Turkey

2 Department of Psychology, Faculty of Science and Letters, Ağr1 İbrahim Çeçen University, Erzurum Yolu 4 Km 04100, Merkez, Ağrı, Turkey

3 Department of Neuroscience, Psychology and Behaviour, University of Leicester, Leicester, UK officially announced (Sahin et al. 2020). After spreading country-wide, on 13 January 2020, the disease crossed Chinese borders and was seen in Thailand. From that time, COVID-19 exponentially spread to more than 185 countries, with the USA, Italy, and Spain being the worst affected countries. The COVID-19 outbreak has forced governments to put strict measures in place to control the virus.

As of 22 May 2020, there were a total of 5,118,416 confirmed cases, 333,212 deaths, and 1,960,223 recovered cases of COVID-19 worldwide (Center for Systems Science and Engineering 2020). Due to the rapidity of the virus spread, on 11 March 2020, it was recognized as the first pandemic derived from the coronavirus family by the World Health Organization (WHO 2020b). While many empirical studies have been carryed out to provide an antiviral vaccine (Mehta et al. 2020), others have been conducted to mostly understand the risk factors associated with high rates of mortality (Zhou et al. 2020). Maintaining mental health plays an important role during the outbreak, and some previous studies showed that 
various psychological and behavioural factors such as COVID-19 severity, self-efficacy and preventive behaviours with mental health were related to physical and mental health (Yıldırım and Güler 2020). Identifying sources of information and characteristics of people who seek information from different outlets are important for policy makers to develop crisis-response strategies. As such, this study endeavoured to identify sources of information and explore the role of various demographic factors such as gender, education level, and perceived socio-economic status on sources of information during the COVID-19 pandemic.

Sources of information during infectious diseases outbreak play a key role to successfully manage public health risk communication. During outbreaks, the public needs to be able to access reliable information that fulfils their needs to engage in protective health behaviours (Vaughan and Tinker 2009; Yildirım et al. 2020). The COVID-19 pandemic can cause uncertainties and requires people to change their behaviours and attitudes towards the pandemic, such as changing their daily news consumption and the ways they receive it. The credibility and usefulness of sources of information related to the pandemic can be useful to direct risk communication channels and messages (Jehn et al. 2011).

With new technological innovations, particularly with regard to cannels of electronic information sources, people tend to largely engage in social networking sites, such as internet journalism and social media, where they can easily access information (Jardine et al. 2015). Reports showed that people from different socioeconomic background tend to access information from different media channels. For example, Internet use has been found to be significantly differing by age group, level of education and income (Statistics Canada 2010). Studies also suggest that there is a strong association between the levels of education and news consumption. In other words, those who are well and formally educated seem to be following the daily news more (Poindexter and McCombs 2001). This connection is also visible in the relationship between news and socioeconomic status although not much is known regarding how much this impact has been altered over the years. For instance, people from lower socioeconomic status do not frequently check daily issues although they do have more perception about what is happening around them (Bergström et al. 2019; Norris and Inglehart 2013). With regard to age, elderly people tend to seek information from their close circle or television (Elareshi 2013).

Due to the nature of the COVID-19 pandemic which requires people to stay at home and practice social distancing, people may prefer to use social networking sites as sources of information because they are instant and interactive platforms. This argument has been documented in previous research, for example, during the H1N1 outbreak and subsequent vaccination program, people predominantly used social networking sites as a source of information (Lam and McGeer 2012).
Identifying how and where people seek information during the COVID-19 outbreak plays an important role for the policy makers to convey the most effective strategies related to controlling the COVID-19 crisis and inform the public about the progress made. As such, the aims of this study were three-fold: (i) to determine the sources of information where the public seek information about COVID-19, (ii) to explore the relationship between demographic factors (e.g. age, gender, education level, socioeconomic status), and sources of information and (iii) to examine the impact of demographic variables on sources of information.

\section{Method}

\section{Participants}

The population of this study included 4624 Turkish speaking individuals between 18 and 89 years of age $(M=30.29$ years old, $\mathrm{SD}=10.97)$. In total, $3236(470 \%)$ of the participants were female and $1388(30 \%)$ were male. In terms of educational level, 500 (10.81\%) were high school graduates or below, 2932 participants $(63.41 \%)$ were university graduates and $1192(25.78 \%)$ held a postgraduate degree. A total of $578(12.50 \%)$ participants perceived their socioeconomic status as being low, $3557(76.92 \%)$ perceived as medium and $489(10.58 \%)$ as high. Concerning marital status, 2698 (58.35\%) participants were single, 1787 (38.65\%) were married and $139(3 \%)$ were widowed/separated.

\section{Measures}

\section{Demographics}

A sociodemographic data form was created to collect demographics. Participants were asked to provide information about their age, gender, education level, marital status and self-reported perceived economic level.

\section{Source of information form}

We asked participants how often they used the following channels as an information source: TV, friends and family, informative meetings, newspapers, internet journalism, and social media. Each source was rated on a 3-point rating scale ranging from 1 (never) to 3 (often). Higher scores represent higher levels of engagement with the relevant source of information.

\section{Procedure}

This study was part of a larger research project examining the impact of the COVID-19 pandemic on psychological health. 
Before completing the study measures, information about the study was presented on the first page of the online survey. Online informed consent was obtained from all participants before entering their demographic information. Those who agreed to collaborate were allowed to proceed to complete the measures which were presented in the same order by the survey software. The average time needed to complete the study was approximately $10 \mathrm{~min}$. All participants voluntarily contributed to the study, and they did not receive any compensation. The study procedure was carried out according to the ethical standards established in the 1964 Declaration of Helsinki and its later amendments. All data collection occurred between 17 March and 1 April 2020.

\section{Data analysis}

SPSS 24.0 for Windows was used for statistical analysis of the data. Descriptive statistics were reported to understand the levels of sources of information among participants. Correlation analysis was performed to test the relationships between demographic variables and sources of information. An independent sample $t$-test was used to compare gender across the sources of information. One-way analysis of variance (ANOVA) was conducted to compare the sources of information by education and economic levels. Skewness and kurtosis statistics were employed to test the assumption of normality, where skewness $<2$ and kurtosis $<7$ represented an acceptable symmetry for a normal univariate distribution.

\section{Results}

\section{Levels of sources of information}

Means and standard deviations regarding the levels of sources of information are reported in Table 1. The most commonly used source of information was internet journalism followed by social media. The least commonly used source of information was newspapers followed by informative meetings.

\section{Correlation between the demographic variables and sources of information}

Correlation results are presented in Table 2. Higher age was correlated with higher levels of watching TV, attending informative meetings and reading newspapers and with lower levels of getting information from friends and family and social media. Females were more likely to get information from their friends and family and social media and less from newspapers. People with higher levels of education were more likely to receive information from informative meetings, newspapers and internet journalism and less likely to receive information from TV. Those who had high levels of perceived economic level tended to report higher levels of receiving information from informative meetings, newspapers and internet journalism.

\section{Gender differences in sources of information}

An independent sample $t$-test was performed to compare the sources of information by gender. The results showed that females obtained information more from their friends and family (2.46 vs 2.16 ) and social media (2.77 vs 2.63$)$ than males, while males reported higher levels of reading newspapers (1.33 vs 1.42$)$ than females. The results are presented in Table 3.

\section{Effects of education level on sources of information}

One-way ANOVA was used to examine the differences in sources of information by the education levels (see Table 4). There were statistically significant differences between group means with respect to TV $(F(2,4623)=6.92, p<0.01)$ and family and friends $(F(2,4623)=5.22, p<0.01)$. Tukey post hoc test revealed that high school graduates or below $(2.29 \pm$ $0.72)$ and university graduates $(2.22 \pm 0.70)$ watched more TV than postgraduates $(2.15 \pm 0.74)$. It also showed that university graduates $(2.39 \pm 0.62)$ obtained more information from their families and friends in comparison to high school graduates or below $(2.31 \pm 0.65)$ and postgraduates $(2.34 \pm 0.63)$.
Table 1 Descriptive statistics regarding sources of information

\begin{tabular}{|c|c|c|c|c|c|c|c|c|}
\hline \multirow[b]{2}{*}{ Information source } & \multirow[b]{2}{*}{ Min } & \multirow[b]{2}{*}{ Max } & \multirow[b]{2}{*}{ Mean } & \multirow[b]{2}{*}{ SD } & \multicolumn{2}{|c|}{ Skewness } & \multicolumn{2}{|l|}{ Kurtosis } \\
\hline & & & & & Statistic & SE & Statistic & SE \\
\hline TV & 1 & 3 & 2.21 & 0.71 & -0.32 & 0.04 & -1.01 & 0.07 \\
\hline Friends and family & 1 & 3 & 2.37 & 0.63 & -0.47 & 0.04 & -0.66 & 0.07 \\
\hline Informative meetings & 1 & 3 & 1.45 & 0.66 & 1.18 & 0.04 & 0.16 & 0.07 \\
\hline Newspapers & 1 & 3 & 1.36 & 0.59 & 1.41 & 0.04 & 0.94 & 0.07 \\
\hline Internet journalism & 1 & 3 & 2.74 & 0.52 & -1.84 & 0.04 & 2.53 & 0.07 \\
\hline Social media & 1 & 3 & 2.73 & 0.54 & -1.87 & 0.04 & 2.54 & 0.07 \\
\hline
\end{tabular}


Table 2 Intercorrelation among the study variables

\begin{tabular}{lllllllllll}
\hline Variable & 1 & 2 & 3 & 4 & 5 & 6 & 7 & 8 & 9 & 10 \\
\hline 1. Age & 1 & & & & & & & & \\
2. Gender & $.36^{* *}$ & 1 & & & & & & & & \\
3. Education level & $.19^{* *}$ & .01 & 1 & & & & & & \\
4. Economic level & $.16^{* *}$ & $.06^{* *}$ & $.15^{* *}$ & 1 & & & & & \\
5. TV & $.14^{* *}$ & -.01 & $-.06^{* *}$ & .01 & 1 & & & & \\
6. Friends and family & $-.21^{* *}$ & $-.22^{* *}$ & -.00 & .00 & $.04^{* *}$ & 1 & & & \\
7. Informative & $.09^{* *}$ & .03 & $.05^{* *}$ & $.05^{* *}$ & $.11^{* *}$ & $.08^{* *}$ & 1 & & & \\
$\quad$ meetings & $.21^{* *}$ & $.07^{* *}$ & $.09^{* *}$ & $.06^{* *}$ & $.13^{* *}$ & .01 & $.23^{* *}$ & 1 & & \\
8. Newspapers & -.00 & .00 & $.06^{* *}$ & $.03^{*}$ & $.09^{* *}$ & $.09^{* * *}$ & $.07^{* *}$ & $.15^{* *}$ & 1 & \\
9. Internet journalism & -.00 & & & & & & \\
10. Social media & $-.18^{* *}$ & $-.12^{* *}$ & .01 & -.02 & .01 & $.21^{* *}$ & -.01 & $-.06^{* *}$ & $.21^{* *}$ & 1 \\
\hline
\end{tabular}

$* * p<0.01 ; * p<0.05$

Because the Levene's $F$ test demonstrated that the homogeneity of variance assumption was not met for informative meetings, newspapers, internet journalism and social media (all $p$ values $<0.01$ ), we addressed this issue by performing the Welch's $F$ test. The results showed a statistically significant effects for all remaining sources of information by education level: informative meetings, Welch's $F(2,1248.49)=6.82$, $p<.001$; newspapers, Welch's $F(2,1270.56)=19.95$, $p<.001$; internet journalism, Welch's $F(2,1196.43)=9.74$, $p<.001$; Welch's $F(2,1203.18)=13.64, p<.001$. The results of Games-Howell post hoc analysis showed that postgraduates $(1.51 \pm 0.68)$ participated more in informative meetings than high school graduates or below $(1.40 \pm 0.67)$ and university graduates $(1.43 \pm 0.64)$. Postgraduates $(1.46 \pm 0.64)$ read more newspapers than high school graduates or below $(1.31 \pm$ $0.55)$ and university graduates $(1.33 \pm 0.57)$. High school graduates or below $(2.62 \pm 0.63)$ obtained less information from internet journalism when compared with university graduates $(2.75 \pm 0.50)$ and postgraduates $(2.75 \pm 0.51)$. They $(2.62 \pm 0.62)$ also engaged in social media less than university graduates $(2.76 \pm 0.52)$ and postgraduates $(2.70 \pm 0.556)$.
University graduates were more active in engaging in social media than postgraduates.

\section{Effects of perceived economic level on sources of information}

One-way ANOVA was performed to examine the differences in sources of information by perceived economic levels (see Table 5). There were statistically significant differences between group means with regard to $\operatorname{TV}(F(2,4623)=5.0$, $p<0.01)$. Tukey post hoc test indicated that those who had a medium economic level $(2.22 \pm 0.71)$ considered TV as a more useful information source than those who had a low economic level $(2.13 \pm 0.74)$. There were no statistically significant differences between group means concerning getting information from friends and families $(F(2,4623)=1.21$, $p>0.05)$ and social media $(F(2,4623)=0.62, p>0.05)$. However, the Levene's $F$ test showed that the homogeneity of variance assumption was violated for informative meetings, newspapers and internet journalism (all $p$ values $<0.05$ ). To correct this, the Welch's $F$ test was therefore utilized. The
Table 3 Independent sample $t$ test results of the comparison between gender with sources of information

\begin{tabular}{llllllll}
\hline Information source & Gender & $\mathrm{N}$ & Mean & $\mathrm{SD}$ & $\mathrm{t}$ & $\mathrm{df}$ & $p$ \\
\hline $\mathrm{TV}$ & Female & 3236 & 2.21 & 0.71 & 0.56 & 4622 & 0.58 \\
& Male & 1388 & 2.20 & 0.72 & & & \\
Friends and family & Female & 3236 & 2.46 & 0.61 & 15.11 & 2608.03 & 0.00 \\
& Male & 1388 & 2.16 & 0.62 & & & \\
Informative meetings & Female & 3236 & 1.43 & 0.66 & -1.95 & 4622 & 0.05 \\
& Male & 1388 & 1.47 & 0.64 & & & \\
Newspapers & Female & 3236 & 1.33 & 0.57 & -4.72 & 2444.91 & 0.00 \\
& Male & 1388 & 1.42 & 0.62 & & & \\
Internet journalism & Female & 3236 & 2.74 & 0.52 & -0.27 & 4622 & 0.78 \\
& Male & 1388 & 2.74 & 0.52 & & & \\
Social media & Female & 3236 & 2.77 & 0.51 & 7.85 & 2272.63 & 0.00 \\
& Male & 1388 & 2.63 & 0.60 & & & \\
\hline
\end{tabular}


Table 4 Means and standard deviations of sources of information by education level

\begin{tabular}{lllll}
\hline Information source & Education level & $\mathrm{N}$ & Mean & $\mathrm{SD}$ \\
\hline TV & High school graduate or below & 500 & 2.29 & 0.72 \\
& University graduate & 2932 & 2.22 & 0.70 \\
& Postgraduate graduate & 1192 & 2.15 & 0.74 \\
Friends family & High school graduate or below & 500 & 2.31 & 0.65 \\
& University graduate & 2932 & 2.39 & 0.62 \\
& Postgraduate graduate & 1192 & 2.34 & 0.64 \\
Informative meetings & High school graduate or below & 500 & 1.40 & 0.67 \\
& University graduate & 2932 & 1.43 & 0.64 \\
& Postgraduate graduate & 1192 & 1.51 & 0.68 \\
Newspapers & High school graduate or below & 500 & 1.31 & 0.54 \\
& University graduate & 2932 & 1.33 & 0.57 \\
Internet journalism & Postgraduate graduate & 1192 & 1.46 & 0.64 \\
& High school graduate or below & 500 & 2.62 & 0.63 \\
& University graduate & 2932 & 2.75 & 0.50 \\
Social media & Postgraduate graduate & 1192 & 2.75 & 0.51 \\
& High school graduate or below & 500 & 2.62 & 0.62 \\
& University graduate & 2932 & 2.76 & 0.52 \\
& Postgraduate graduate & 1192 & 2.70 & 0.56 \\
\hline
\end{tabular}

analysis demonstrated a statistically significant effect for informative meetings, Welch's $F(2,888.73)=5.07, p<.001$ and newspapers, Welch's $F(2,888.29)=8.85, p<.001$, but not for internet journalism, Welch's $F(2,884.71)=2.29$, $p>.005$. An analysis of post hoc test as determined by

Table 5 Means and standard deviations of sources of information by economic level

\begin{tabular}{lllll}
\hline Information source & Economic level & $\mathrm{N}$ & Mean & $\mathrm{SD}$ \\
\hline TV & Low & 578 & 2.13 & 0.74 \\
& Medium & 3557 & 2.22 & 0.71 \\
& High & 489 & 2.16 & 0.74 \\
Friends and family & Low & 578 & 2.34 & 0.63 \\
& Medium & 3557 & 2.37 & 0.63 \\
& High & 489 & 2.34 & 0.64 \\
Informative meetings & Low & 578 & 1.37 & 0.66 \\
& Medium & 3557 & 1.45 & 0.65 \\
& High & 489 & 1.50 & 0.67 \\
Newspapers & Low & 578 & 1.30 & 0.55 \\
& Medium & 3557 & 1.36 & 0.59 \\
Internet journalism & High & 489 & 1.45 & 0.65 \\
& Low & 578 & 2.71 & 0.55 \\
& Medium & 3557 & 2.74 & 0.51 \\
Social media & High & 489 & 2.78 & 0.51 \\
& Low & 578 & 2.74 & 0.55 \\
& Medium & 3557 & 2.73 & 0.54 \\
& High & 489 & 2.70 & 0.54 \\
\hline
\end{tabular}

Games-Howell revealed that people with low economic level $(1.37 \pm 0.66)$ attended informative meetings less compared to people with medium $(1.45 \pm 0.65)$ and high $(1.50 \pm 0.67)$ socioeconomic status. Similarly, people with a high economic level $(1.45 \pm 0.65)$ read more newspapers than those with medium $(1.36 \pm 0.59)$ and low $(1.30 \pm 0.55)$ economic levels. The mean differences between medium and low socioeconomic status were also statistically significant.

\section{Discussion}

To the best of our knowledge, this is the first study identifying sources of information and examining the impact of demographic factors on sources of information during the early phase of the COVID-19 outbreak in a large sample drawn from the general public in Turkey. The results showed that the most preferable source of information was rated as internet journalism and social media. This result is expected since internet and social networking sites are widely used globally. With easy access to technological devices such as phone and iPad, receiving information from internet journalism and social media can be more convenient and cost effective for people during the pandemic. Sources of information during public health crisis can play a key role in protecting mental health if used efficiently. The Mental Health Commission of Canada (2020) underscored that it is critical to maintain positive mental health by carefully choosing credible sources of information during the COVID-19 outbreak. 
The results also showed that higher age, educational attainment and economic status were related to higher levels of seeking information from TV, newspapers, internet journalism and informative meetings. These results are partly consistent with previous findings. Younger people were found to be more comfortable using internet as a source of information. People with lower education attainment may lack in knowledge of or receive information from virtual platforms such as internet journalism (Ramsey et al. 2009). The differences in the results of this study and those of previous studies are indeed expected due to the nature of COVID-19 which requires all people from different socioeconomic backgrounds to stay at home. Thus, it is possible that they use various sources of information (e.g. TV and internet journalism) for crossvalidation of the credibility of the information during the pandemic.

With regard to gender differences, females reported that they mainly received information from their friends and family and social media compared with males. This can be related to cultural background where Turkish women typically gather at homes and discuss everyday matters (Tuncer 2018). Although the COVID-19 pandemic imposes people to stay at home, it is plausible to assume that Turkish women can still receive information from their families and friends over the phone or other communication tools. However, the accuracy of information from such sources can be an issue of dispute as studies suggest that people with better access to information through mass communication tools receive more favourable content (Strömberg 2004).

Concerning the education level, we found that participants significantly differ in seeking information from different sources. While those with low education attainment (e.g. high school graduate or below) reported that they sought information mainly from traditional media such as TV, highly educated people received information from relatively contemporary sources of information (e.g. internet journalism, informative meeting). These results demonstrate that those who have higher education level are more familiar with digital media technologies and prefer receiving information from online sources. Although misinformation is highly likely on online platforms, and new(s) media literacy should carefully be considered at this level too, because online news websites deliver an unceasing stream of information, provide instantly updated content, clear infographics, leisure and cultural products based on the understanding of readers' needs, people are more likely to apply them for the lastest updates.

With respect to socioeconomic status, seeking information from TV and newspapers were the most common sources of information among people with perceived medium and high socioeconomic status, whereas the least reported source of information was attending informative meetings among people with perceived low socioeconomic status. Previous research suggests that social inequalities shape the forms of news consumption decisions individuals take regarding news (Lindell 2017). Therefore, social inequality in news consumption and sources may not only be about the number of news sources an audience used but also a matter of how people receive their news and what mediums they rely on.

This study provides evidence that the source of information is significantly influenced by demographic factors. Academics, health officials and policy makers can consider the results of this study to develop effective crisis-response and health communication strategies in the light of variations in the demographic factors. Given that the differences in the ways for receiving the news and consuming it may be shaped by sociodemographic factors, awareness about how people acquire information can also help policy makers to inform the public and deliver reliable information about the pandemic in a faster and more convenient way.

This study has two main strengths. First, the data collection was held between 17 March and 1 April 2020 following the announcement of Turkey's outbreak on 17 March 2020. This early start enables us to timely assess where the Turkish community receives information and how this differs based on demographic factors. Second, a large number of participants from different socioeconomic backgrounds could help to generalize the present findings to a wider population.

It is important to acknowledge that the current research has several drawbacks that need to be addressed in future studies. The first limitation was the use of an online survey which may carry biases. Those who were unable to use the internet or have limited access were not satisfactorily represented in this investigation. Nonetheless, collecting data via an online survey appeared to be the most realistic and feasible method during the COVID-19 pandemic. Second, there were only a limited number of variables examined in this study. Future research should consider how people are influenced by the news content and how this varies in different sources of information. Future studies should also specifically look at how the use of digital and portable news media vary according to demographics, socioeconomic and educational factors which will again allow policymakers to develop effective health communication strategies especially in times of crisis.

In sum, internet journalism and social media were the most preferable source of information. Age, educational attainment and economic level were related to sources of information. There are significant variations in gender, education level and economic status in seeking information from different sources. These results would facilitate development of effective crisis-response strategies during the pandemic. 
Acknowledgements We thank all participants who voluntarily contributed to this study.

Funding The authors received no financial support for the research, authorship, and/or publication of this article.

\section{Compliance with ethical standards}

Conflicts of interest The authors declared no conflicts of interest with respect to the research, authorship, and/or publication of this article.

Ethical approval All procedures performed in studies involving human participants were in accordance with the ethical standards of the institutional and/or national research committee and with the 1964 Helsinki declaration and its later amendments or comparable ethical standards.

Informed consent Consent was obtained from all participants included in the study.

\section{References}

Bergström A, Strömbäck J, Arkhede S (2019) Towards rising inequalities in newspaper and television news consumption? A longitudinal analysis, 2000-2016. Eur J Commun 34(2):175-189. https://doi. org/10.1177/0267323119830048

Center for Systems Science and Engineering (2020) Coronavirus COVID-19 global cases at Johns Hopkins University. https:// coronavirus.jhu.edu/map.html. Accessed 22 May 2020

Elareshi M (2013) News consumption in Libya. Cambridge Scholars, Newcastle

Jardine CG, Boerner FU, Boyd AD, Driedger SM (2015) The more the better? A comparison of the information sources used by the public during two infectious disease outbreaks. PLoS One 10(10):1-18. https://doi.org/10.1371/journal.pone.0140028

Jehn M, Kim Y, Bradley B, Lant T (2011) Community knowledge, risk perception, and preparedness for the 2009 influenza a/H1N1 pandemic. J Public Health Manag Pract 17(5):431-438. https://doi.org/ 10.1097/PHH.0b013e3182113921

Lam PP, McGeer A (2012) Communications Strategies for the 2009 Influenza A (H1N1) Pandemic. National Collaborating Centre for Infectious Diseases. https://nccid.ca/publications/communicationstrategies-for-the-2009-influenza-a-h1n1-pandemic/. Accessed 22 July 2020

Lindell J (2017) Distinction recapped: digital news repertoires in the class structure. New Media Soc 20(8):3029-3049. https://doi.org/10. $1177 / 1461444817739622$

Mehta P, McAuley D, Brown M, Sanchez E, Tattersal R (2020) COVID19: consider cytokine storm syndromes and immunosuppression. Lancet 395(10229):1033-1035. https://doi.org/10.1016/S0140$6736(20) 30628-0$
Mental Health Commission of Canada (2020) Choosing sources of information carefully is critical to COVID-19 mental well-being says Mental Health Commission of Canada. https://www. mentalhealthcommission.ca/English/news-article/13920/choosingsources-information-carefully-critical-covid-19-mental-well-beingsays. Accessed 22 July 2020

Norris P, Inglehart R (2013) Digital divide. In: Towse R \& Christian H (ed) Handbook on the digital creative economy. Edward Elgar, Northampton, pp 90-104

Poindexter PM, McCombs ME (2001) Revisiting the civic duty to keep informed in the new media environment. J Mass Commun Q 78: $113-126$

Ramsey SD, Zeliadt SB, Arora NK et al (2009) Access to information sources and treatment considerations among men with local stage prostate cancer. Urology 74(3):509-515. https://doi.org/10.1016/j. urology.2009.01.090

Sahin AR, Erdogan A, Agaoglu PM, Dineri Y, Çakırcı AY, Senel ME (2020) 2019 novel coronavirus (COVID-19) outbreak: a review of the current literature. Eurasian J Med Oncol 4(1):1-7. https://doi. org/10.14744/ejmo.2020.12220

Statistics Canada (2010) Internet use by individuals, by selected characteristics. https://www150.statcan.gc.ca/t1/tb11/en/tv.action?pid= 2210001101. Accessed 22 July 2020

Strömberg D (2004) Radio's impact on public spending. Q J Econ 119: 189-221. https://doi.org/10.1162/003355304772839560

Tuncer S (2018) Women and public space in Turkey: gender, modernity and the urban experience. IB Tauris, London

Vaughan E, Tinker T (2009) Influenza preparedness and response for vulnerable populations. Am J Public Health 99(S2):331-332

World Health Organization (2020a) Novel Coronavirus (2019-nCoV) Situation Report-1 - 21 January 2020. https://www.who.int/docs/ default-source/coronaviruse/situation-reports/20200121-sitrep-12019-ncov.pdf?sfvrsn=20a99c10_4. Accessed 22 July 2020

World Health Organization (2020b) WHO Director-General's opening remarks at the media briefing on COVID-19 - 11 March 2020. https://www.who.int/dg/speeches/detail/who-director-general-sopening-remarks-at-the-media-briefing-on-covid-19\%2D\%2D-11march-2020. Accessed 22 July 2020

Yıldırım M, Geçer E, Akgül, Ö (2020) The impacts of vulnerability, perceived risk, and fear on preventive behaviours against COVID19. Psychology, Health \& Medicine 1-9. https://doi.org/10.1080/ 13548506.2020.1776891

Yıldırım M, Güler A (2020) COVID-19 severity, self-efficacy, knowledge, preventive behaviors, and mental health in Turkey. Death Stud 1-8. https://doi.org/10.1080/07481187.2020.1793434

Zhou F, Yu T, Du R et al (2020) Clinical course and risk factors for mortality of adult inpatients with COVID-19 in Wuhan, China: a retrospective cohort study. Lancet 395(10229):1054-1062. https:// doi.org/10.1016/S0140-6736(20)30566-3

Publisher's note Springer Nature remains neutral with regard to jurisdictional claims in published maps and institutional affiliations. 